\title{
Report on symposium on the surveillance and control of sexually transmitted diseases
}

\author{
VIENNA, 21-25 SEPTEMBER 1976
}

The symposium was attended by 41 participants from 20 countries including two representatives of the International Union against the Venereal Diseases and the Treponematoses and one representative of the International Planned Parenthood Federation. The participants represented a wide range of disciplines including epidemiologists, laboratory experts, sociologists, health educators, economists, venereologists, and health administrators. The purpose of the symposium was to examine those aspects of the control of sexually transmitted diseases (STDs) which apply to Europe.

Chosen topics for the meeting were:

1. extent and trends of the sexually transmitted diseases;

2. case finding by (a) screening; (b) contact tracing;

3. economic aspects;

4. health education;

5. training of personnel.

The principal conclusions and recommendations may be summarised as follows.

\section{Extent and trends}

In view of reports from many countries indicating an increase in the number of cases of all of the sexually transmitted diseases since the 1971 meeting in Copenhagen (WHO, 1972), a great effort by all disciplines will be required if these diseases are to be controlled. Furthermore, the co-operation between WHO and all international groups concerned directly or indirectly with the control of sexually transmitted diseases should be strengthened.

Changes in existing legislation on the reporting of the sexually transmitted diseases for statistical purposes or for contact tracing should be considered in the light of all of the STDs now being treated in the clinics.

A unified classification based on specified diagnostic criteria for statistical reporting should be established. In addition, national statistics relating at least to primary and secondary syphilis and gonorrhoea should be collected by age groups based on the structure defined in the International Classification of Diseases. Age specific attack rates can then be calculated.
A draft form for this purpose was prepared at the meeting with the recommendation that it be considered by National Committees for the Classification of Diseases and Deaths and by the next STD expert committee or similar headquarters meeting, and be placed on the agenda of the WHO Committee for International Disease Classification as soon as practicable.

Continuing efforts should be made to encourage general practitioners to involve themselves more closely in the venereal disease programme.

\section{Case finding}

\section{SCREENING}

Having reviewed in detail the methods available for the screening of STD, (and having seen the facilities for screening prostitutes in Vienna), the symposium concluded that case finding applied to the whole sexually-active population is extremely costly when the prevalence of the disease is low. Such programmes should therefore be undertaken only among groups at high risk.

Mass screening is only effective for diseases where blood samples can be examined using uncomplicated inexpensive methods. It is most cost/effective if it is combined with investigations for other conditions using automated techniques.

Screening for gonorrhoea, for which (in the absence of an effective serological test) cultures are necessary, is most feasible in groups of patients who are examined for other reasons (for example, gynaecological), and this procedure should be encouraged.

In screening for syphilis the VDRL and the TPHA tests, both of which can be automated, were recommended as the methods of choice. The VDRL method is cheaper.

It is important that the antibiotic sensitivities of gonococci be continually monitored in all countries so that treatment is soundly based.

This is particularly necessary following recent alarming reports of the sudden appearance in a number of countries (including one in Europe) of penicillinase-producing gonococci which are resistant to penicillin and to streptomycin. This might in time involve other antibiotics also. All European 
countries should be asked to send any such strains which may be encountered to the WHO Reference Laboratory at the State Serum Institute, Copenhagen, so that multiple sensitivity tests and other special studies may be undertaken. Furthermore, WHO headquarters at Geneva should be notified immediately so that anticipated future developments in this field can be monitored.

\section{CONTACT TRACING}

Contact interviewers are best employed working in clinics in conjunction with other STD workers, and the maximum use of 'voluntary persuasion by contact slips' should be made. In very small clinics this may be the only feasible method.

Whatever the type of personnel recruited and trained as 'contact interviewers', they should be responsible to the physician in charge.

Contact tracing is more successful in cases of primary and secondary syphilis than in cases with gonorrhoea.

Since an adequate form for use in international contact tracing was proposed to countries of the region during the 1971 Working Group on the Intercountry Spread of Venereal Diseases, participants in the present symposium recommended that a further appraisal of the use and effectiveness of this procedure be undertaken soon.

Preferring the term 'rapid simultaneous' to 'epidemiological' treatment, most participants agreed about its usefulness and efficacy: (a) in countries or clinics lacking adequate diagnostic facilities; (b) in many practical individual situations, even in wellequipped and adequately staffed premises. The increased use of this type of treatment was recommended, especially in higher risk groups, for primary and secondary syphilis, gonorrhoea, and other STDs where appropriate, even in countries well equipped and well staffed in terms of STD control facilities.

As the inhibitory effects of commercially available vaginal spermicides on the growth of several infectious agents have been effectively demonstrated, and as their acceptability by a high proportion of women has been shown in field trials which are giving some promising results, further control studies of this acceptability and effectiveness should be made among high-risk groups in Europe.

\section{Economic aspects}

Health economics has become a necessity because of spiralling health costs, but in only a few countries have any assessments been made of the importance of the STD to the economy of their health sector and to their general economy.
A multinational study on the cost aspects of the STD should be carried out, not only of gonorrhoea and syphilis, but of other STDs as well. Such studies are useful in negotiations about funds and resources for the control of these diseases.

Such studies should also cover the cost/efficiency and cost/effectiveness of the various methods of case control-for example, finding, treatment, health education, prophylaxis. Furthermore, an attempt should be made to discover in what circumstances and with which degree of emphasis the individual components may best be applied.

Any study relating to economic aspects in the field of STD must include investigators from various disciplines, at least a health economist and an STD specialist.

Starting in 1977 after consultation with health economists and STD experts in the region, the WHO Regional Office for Europe should provide a forum for establishing a standardised protocol for a multinational study which should be co-ordinated within its programme on economic aspects of communicable diseases.

\section{Health education}

Greater use should be made of the important tool of health education, involving health workers and teachers and the general public in all fields to ensure the maximum benefit from existing methods of control among infected or potentially infected persons.

The STD education programmes should be tailored to each target group, taking into account their cultural and socioeconomic conditions as well as the accessibility, acceptability and even the attractiveness of the existing STD services. Collaboration between authorities is required (such as those responsible for education, health care, care of migrant workers, seafarers, occupational health, etc.).

Pilot programmes on health education integrated in existing STD services should be initiated in a number of WHO member states in Europe. Such programmes should be co-ordinated by WHO and supported by the International Union against the Venereal Diseases and the Treponematoses, by the International Union for Health Education, and by the International Labour Office.

\section{Training of personnel}

There is an urgent need for better multidisciplinary training for physicians as well as for nurses and auxiliary health personnel, with greater emphasis on epidemiology, psychology, health education, mental health, and human sexual behaviour. 
The undergraduate curriculum should not only comprise the clinical, diagnostic, and treatment aspects of the STD but also basic human sexual behaviour characteristics, including variant behaviour, in the context of human sexuality as a whole.

The training of future specialists should provide more detailed studies on the clinical, diagnostic, and treatment aspects; the epidemiology of the STD and up-to-date control methods; skills in communicating with the patient about sexual matters; co-operation with paramedical personnel; and a working association with those in other disciplines.

Continuing medical education should involve not only specialists but all health personnel involved in the control and treatment of the STD (especially general practitioners) by means of full-time courses, seminars and meetings, journals, and other methods of communication.

The WHO should consider preparing a simple guide to the diagnosis, treatment and management of the STDs, intended primarily for general practitioners so that they may more effectively contribute to their control by managing more efficiently the many cases they encounter.

\section{Reference}

WHO Regional Office for Europe (1972). The Inter-Country Spread of Venereal Diseases: Report on a Working Group (document EURO 1101). Copenhagen. 